\title{
Antidepressant discontinuation syndrome
}

\author{
Matthew Gabriel, Verinder Sharma MBBS
}

Cite as: CMAJ 2017 May 29;189:E747. doi: 10.1503/cmaj.160991

\section{1}

\section{Antidepressant discontinuation syndrome is common}

About $20 \%$ of patients develop antidepressant discontinuation syndrome following an abrupt stoppage of or marked reduction in the dose of an antidepressant taken continuously for one month. ${ }^{1}$ Symptoms are usually mild and may occur following treatment with any type of antidepressant. Symptoms occur within two to four days after drug cessation and usually last one to two weeks (occasionally may persist up to one year). If the same or a similar drug is started, the symptoms will resolve within one to three days. Sociodemographic and clinical factors associated with increased vulnerability have not been identified. ${ }^{2}$ Among the serotonin reuptake inhibitors, paroxetine is associated with the highest incidence of the syndrome and fluoxetine with the lowest. Because of venlafazine's short half-life, the syndrome may occur more frequently following cessation and symptoms may be more severe. ${ }^{1}$

\section{Symptoms are vague and variable}

Failure to recognize antidepressant discontinuation syndrome may result in medical or psychiatric misdiagnosis. The mnemonic FINISH summarizes the symptoms of antidepressant discontinuation syndrome: Flu-like symptoms (lethargy, fatigue, headache, achiness, sweating), Insomnia (with vivid dreams or nightmares), Nausea (sometimes vomiting), Imbalance (dizziness, vertigo, light-headedness), Sensory disturbances ("burning," "tingling," "electric-like" or "shock-like" sensations) and Hyperarousal (anxiety, irritability, agitation, aggression, mania, jerkiness). ${ }^{3}$

\section{3}

\section{The syndrome needs to be distinguished from relapse}

Cessation of antidepressant therapy may increase the risk of relapse of depression or anxiety. Unlike the symptoms of antidepressant discontinuation syndrome, symptoms of relapse usually take more than a few days to appear and to disappear following reintroduction of the antidepressant. ${ }^{4}$

\section{Patient education may reduce risk}

Physicians should be vigilant at times when patients may consider stopping an antidepressant (e.g., during pregnancy). Because not all formulations of the same drug are bioequivalent, there may be an unintended reduction in drug concentration if an antidepressant is switched to another formulation. To minimize risk of the syndrome, patients should be encouraged to consult their physician before stopping an antidepressant. Prescribing an antidepressant with a longer half-life or tapering the medication over six to eight weeks may reduce the risk. ${ }^{4}$

\section{5} Treatment of the syndrome needs to be individualized

Management of antidepressant discontinuation syndrome needs to be done on an individual basis because of a lack of specific treatment data (Box 1). 2,3,5
Box 1: Prevention and management of antidepressant discontinuation syndrome $\mathrm{e}^{2,3,5}$

- Tapering may not be necessary for patients taking an antidepressant for less than four weeks or those who are taking fluoxetine.

- Faster tapering may be possible in cases where doses are low.

- Antidepressants with short half-lives need to be tapered gradually; however, gradual tapering may not prevent the syndrome in all cases.

- Patients should be reassured that symptoms are reversible, not life-threatening and usually selflimiting.

- Switching to fluoxetine when considering stopping another antidepressant may be helpful in some cases.

- If symptoms are severe, the drug should be reintroduced and a slower taper started.

\section{References}

1. Diagnostic and statistical manual of mental disorders. 5th ed. Arlington (VA): American Psychiatric Association; 2013:712-4.

2. Fava GA, Gatti A, Belaise C, et al. Withdrawal symptoms after selective serotonin reuptake inhibitor discontinuation: a systematic review. Psychother Psychosom 2015;84:72-81.

3. Berber MJ. FINISH: remembering the discontinuation syndrome. Flu-like symptoms, insomnia, nausea, imbalance, sensory disturbances, and hyperarousal (anxiety/agitation). J Clin Psychiatry 1998;59:255.

4. Warner $\mathrm{CH}$, Bobo W, Warner C, et al. Antidepressant discontinuation syndrome. Am Fam Physician 2006;74:449-56.

5. Ogle NR, Akkerman S. Guidance for the discontinuation or switching of antidepressant therapies in adults. J Pharm Pract 2013;26:389-96.

Competing interests: Verinder Sharma has received grants from Assurex, Genome Canada, Neurocrine Biosciences, Stanley Medical Research Institute and Sunovion Pharmaceuticals. No other competing interests were declared.

This article has been peer reviewed.

Affiliations: London Regional Mental Health Care (Sharma), London, Ont.; University of Ottawa (Gabriel), Ottawa, Ont.

Correspondence to: Matthew Gabriel, mgabr041@ uottawa.ca 